Gefässchirurgie $2013 \cdot 18: 618-620$

DOI 10.1007/s00772-013-1218-5

Online publiziert: 2. November 2013

(c) Springer-Verlag Berlin Heidelberg 2013

\section{H.-H. Eckstein}

Klinik und Poliklinik für Vaskuläre und Endovaskuläre Chirurgie, Klinikum rechts der Isar der Technischen Universität München

\title{
Weitere randomisierte Studien zur asymptomatischen Karotisstenose - ein Muss!
}

Liebe Kolleginnen und Kollegen,

das Leitthema dieser Ausgabe der Gefässchirurgie gibt Ihnen einen Überblick über die aktuell laufenden randomisierten Studien (RCTs) zur asymptomatischen Karotisstenose. Von besonderem Interesse ist dabei die erstmals publizierte Protokolländerung der SPACE-2-Studie, die zuerst in der Gefässchirurgie erscheint und danach auch im Nervenarzt und in der Clinical Neuroradiology abgedruckt werden wird. Zusammen mit den Übersichten zu ACST-2, ECST-2 und CABACS erhalten Sie einen umfassenden Überblick $\mathrm{zu}$ allen laufenden europäischen RCTs.

In den USA wurde kürzlich die CREST-2-Studie zur asymptomatischen Karotisstenose durch das „National Institute of Neurological Disorders and Stroke (NINDS)“ zur Förderung freigegeben. Ähnlich wie im neuen Studiendesign von SPACE-2 werden in zwei parallel laufenden Studien die Karotis-TEA (CEA) und das Karotisstenting (CAS) mit einem "best medical treatment (BMT)" verglichen. Das „US Center for Medicare and Medicaid Services" hat daher beschlossen, CAS nicht als „Kassenleistung " freizugeben. Im Übrigen wurde der in CREST-1 so heftig kritisierte kombinierte primäre Endpunkt „Schlaganfall, Tod oder Myokardinfarkt" nun auch in CREST-2 zugunsten des klassischen primären Endpunkts "Schlaganfall oder Tod“ fallen gelassen. Dies bedeutet nicht, dass eine periprozedurale Myokardischämie keine potenziell schwere Komplikation darstellen würde. Im Gegenteil, periprozedurale Myokardinfarkte müssen weiterhin als sekundäre Endpunkte erfasst werden, als primärer Endpunkt sind sie in einer „Karotisstudie " jedoch ungeeignet. Sehr wichtig ist darüber hinaus, dass sich alle laufenden RCTs bereit erklärt haben, gemeinsame Datenauswertungen und weitere wissenschaftlich interessante Substudien (s. unten) durchzuführen.

Basierend auf den großen RCTs zur asymptomatischen Karotisstenose aus den 1990er-Jahren (ACST, ACAS) wird in der aktuellen deutsch-österreichischen S3-Leitlinie eine starke Empfehlung ausgesprochen, beim Vorliegen einer 60-99\%igen Karotisstenose (NASCET-Kriterien) die CEA zu erwägen, da hierdurch das Schlaganfallrisiko gering, aber statistisch signifikant reduziert wird. Voraussetzung hierfür ist allerdings die Einhaltung einer perioperativen Schlaganfallrate/Letalität von $<3 \%$. CAS kann als Alternative erwogen werden, sofern ebenfalls eine Komplikationsrate von $<3 \%$ nachweislich eingehalten wird (• Tab. 1). Die S3-Leitlinie weist allerdings auch darauf hin, dass diese Empfehlungen auf Studien beruhen, die z.T. in den 90er-Jahren des letzten Jahrhunderts durchgeführt worden sind und sich die medikamentösen Therapieoptionen seither signifikant verbessert haben. Da andererseits auch die Komplikationsraten von CEA und CAS über die Jahre gesenkt werden konnten, erscheinen neue Headto-head-Studien zwischen allen drei Modalitäten zwingend geboten [3-5].

Selbstverständlich gibt es auch Kritik und Skepsis zu weiteren randomisierten Karotisstudien. Die Diskussion betrifft hierbei folgende Problembereiche:
1. Es wird darauf hingewiesen, dass der schlaganfallpräventive Effekt der CEA für hochgradige Karotisstenosen auf höchstem Evidenzlevel bewiesen wurde. Warum soll dies nun wieder "ohne Not" infrage gestellt werden, zumal die CEA (übrigens auch CAS) im DSRG-System gut abgebildet wird? Der Einwand ist nachvollziehbar und muss ernst genommen werden. Schließlich erleiden allein in Deutschland mindestens $20.000 \mathrm{~Pa}$ tienten/Jahr einen karotisassoziierten Schlaganfall. In den meisten Fällen lag dabei eine zuvor asymptomatische Karotisstenose vor. Andererseits liegt das Risiko einer zerebralen Ischämie nur zwischen 1 und 5\%/Jahr und auch unter den Bedingungen von ACAS und ACST mussten 1520 CEAs durchgeführt werden, um einen karotisbedingten Schlaganfall in 5 Jahren zu vermeiden. Zusätzlich wird insbesondere von neurologischer Seite darauf hingewiesen, dass ein konsequentes BMT verbunden mit einer intensiven „Life-styleBeratung“ (u. a. Nikotinverzicht, Bewegung, mediterrane Kost) das Risiko einer karotisassoziierten zerebralen Ischämie teilweise auf 0,5\%/Jahr verringern kann [1,2]. Diese „Botschaft" führt schon jetzt dazu, dass die Indikation zur CEA bei diesen Patienten immer häufiger kritisch hinterfragt wird, bzw. diese Patienten gar nicht mehr zugewiesen werden. Die Situation erinnert aber auch ein wenig an die 1970er- und 1980er-Jahre, in denen die CEA als gefährliche und 
Tab. 1 Empfehlungen zur asymptomatischen Karotisstenose. (Adaptiert nach [5])

\begin{tabular}{|c|c|c|c|}
\hline Nr. & Text der Empfehlung & Grad $^{a}$ & LoEb \\
\hline A & $\begin{array}{l}\text { Patienten sollen vor und nach einer CEA ASS einnehmen, eine } \\
\text { Dauertherapie mit ASS soll nicht unterbrochen werden }\end{array}$ & $\uparrow \uparrow$ & 1 \\
\hline B & $\begin{array}{l}\text { Die medikamentöse und nichtmedikamentöse Basistherapie zur Verhin- } \\
\text { derung arteriosklerotischer Komplikationen soll sich an den entsprechen- } \\
\text { den LL und Standards orientieren (Lipidstoffwechsel, antihypertensive } \\
\text { Therapie und Diabetes-mellitus-Therapie, „lifestyle modification“) }\end{array}$ & $\uparrow \uparrow$ & 1 \\
\hline C & $\begin{array}{l}\text { Die CEA soll bei Patienten mit einer 60-99\%igen asymptomatischen } \\
\text { Karotisstenose erwogen werden, da das Schlaganfallrisiko bei diesen } \\
\text { Individuen gering, aber statistisch signifikant reduziert wird }\end{array}$ & $\uparrow \uparrow$ & 1 \\
\hline D & $\begin{array}{l}\text { Der Nutzen der CEA bei Patienten mit asymptomatischer Karotisstenose } \\
\text { besteht nur, wenn die Behandlung mit einer Komplikationsrate von } \\
\text { weniger als 3\% durchgeführt wird }\end{array}$ & $\uparrow \uparrow$ & 1 \\
\hline$E$ & $\begin{array}{l}\text { Der Nutzen der CEA bei Patienten mit asymptomatischer Karotisstenose } \\
\text { besteht vor allem für Männer und Patienten mit einer Lebenserwartung } \\
\text { von mindestens } 5 \text { Jahren }\end{array}$ & $\uparrow$ & 1 \\
\hline $\mathrm{F}$ & $\begin{array}{l}\text { Der Stellenwert der Therapieverfahren (CEA, CAS, BMT) bei asymptomati- } \\
\text { scher Karotisstenose sollte in kontrollierten Studien überprüft werden }\end{array}$ & GCP & - \\
\hline G & $\begin{array}{l}\text { Wenn bei einer asymptomatischen Karotisstenose die Indikation zur in- } \\
\text { vasiven Behandlung besteht, kann CAS alternativ erwogen werden, wenn } \\
\text { das behandelnde Zentrum zur CEA analoge Qualitätskriterien mit einer } \\
\text { Komplikationsrate von weniger als 3\% nachweislich einhält }\end{array}$ & $\leftrightarrow$ & $2 b$ \\
\hline $\mathrm{H}$ & $\begin{array}{l}\text { Bei erschwerten Bedingungen für eine CEA kann bei bestehender } \\
\text { Therapieindikation in Zentren mit nachgewiesener Komplikationsrate }<3 \% \\
\text { alternativ eine CAS erwogen werden }\end{array}$ & $\leftrightarrow$ & $2 b$ \\
\hline \multicolumn{4}{|c|}{ 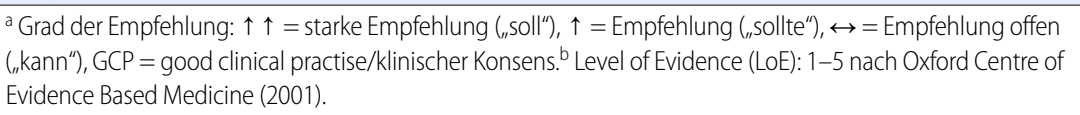 } \\
\hline
\end{tabular}

unnütze Behandlung in Frage gestellt worden war. Die daraufhin durchgeführten RCTs zur symptomatischen Stenose (NASCET, ECST u. a.) haben dann z. T. sehr rasch bewiesen, dass die CEA in der Sekundärprävention der karotisbedingten zerebralen Ischämie unverzichtbar ist. Nur zur Erinnerung: NASCET wurde aufgrund der hochsignifikanten Überlegenheit der CEA für 70-99\%ige Stenosen aus ethischen Gründen abgebrochen! Diese Erfahrung sollte uns ermutigen, auch im 20. Jahrhundert „das Wagnis einzugehen“, die CEA mit BMT und CAS im direkten Vergleich zu untersuchen.

2. Es kann davon ausgegangen werden, dass auch nach erfolgreicher Durchführung dieser RCTs vermutlich nur ein kleinerer Teil der Patienten wirklich einer Revaskularisierung bedarf bzw. auch maximal konservativ behandelte Patienten einen karotisassoziierten Schlaganfall erleiden werden, und dies auch dann, wenn aufgrund positiver Studienergebnisse auf jede revaskularisierende Maßnahme verzichtet wurde. Die offene und wissenschaftlich spannendste Frage betrifft also die Detektion von Patienten mit instabilen und potenziell embolisierenden Karotisstenosen und damit die Entwicklung einer individuell zugeschnittenen Therapie. Um diesem „hehren Ziel“ näher zu kommen, haben sich nun alle großen RCTs bereit erklärt, gemeinsame Substudien zu behandelnde Themen betreffen z. B. die Validität der mittels MRT ermittelten Plaquemorphologie, die klinische Bedeutung von periprozeduralen Mikroembolien oder auch der klinische Nutzen von seriellen Biomarkerbestimmungen. Es ist zu fordern, dass derartige gut projektierte studienübergreifende Substudien gelingen, um das individuelle karotisassoziierte Schlaganfallrisiko in nicht zu ferner Zukunft evidenzbasiert abschätzen zu können.

3. Erfahrungsgemäß werden maximal 10-20\% aller potenziellen Studienpatienten tatsächlich für den betreffenden RCT rekrutiert, das Ergebnis der durchzuführen. Mögliche prospektiv
Studie dann aber gern auf $100 \%$ der Patienten übertragen. Dieses Problem, von Statistikern als fehlende „externe Validität“ bezeichnet, begleitet RCTs immer dann, wenn die zu testenden Verfahren (wie z. B. CAS) „frei verfügbar" sind und seitens der Krankenkassen auch finanziert werden. Wie bereits erwähnt, ist in vielen Ländern (z. B. USA, Frankreich etc.) der Zugang zu CAS für die meisten Patienten nur im Rahmen von Studien möglich! Zumindest in Deutschland ist die Freiheit diesbezüglich grenzenlos - ob das wirklich gut ist, steht auf einem anderen Blatt. Um die Aussagekraft der laufenden RCTs zu erhöhen, ist es aber zweifelsfrei notwendig, möglichst viele Patienten in SPACE-2 oder eine der anderen RCTs zu rekrutieren. Nur so kann es gelingen, wirklich repräsentative Ergebnisse zu ermitteln. Hier ist jede(r) Gefäßchirurg(in) aufgefordert, einen kleinen aber relevanten Teil zum Erkenntnisgewinn in der Gefäßmedizin beizutragen. Nochmals: Es waren die großen (leider kaum im deutschsprachigen Raum) durchgeführten RCTs, die unser Fach vorangebracht haben!

4. Insbesondere die SPACE-2-Studie wird kritisch hinterfragt, da das neue Protokoll nur noch den randomisierten Vergleich zwischen CEA+BMT vs. BMT (SPACE-2a) und CAS+BMT vs. BMT (SPACE-2b) vorsieht. Die für viele Gefäßchirurg(inn)en brennende Frage nach der Über- oder Unterlegenheit von CAS vs. CEA bleibt dabei ausgeklammert. Es ist leider eine Tatsache, dass SPACE-2 innerhalb von mehr als 3 Jahren nur etwa $400 \mathrm{~Pa}$ tienten randomisiert hat. Für die Rekrutierung der geplanten $3600 \mathrm{~Pa}$ tienten würde man noch etwa $30 \mathrm{Jah}$ re benötigt, eine absurde Vorstellung. Ganz offensichtlich verunsichert eine dreiarmige RCT Patienten, zuweisende Kollegen und aufklärende Ärzte gleichermaßen. Es mag sein, dass ein dreiarmiger RCT in einer pharmakologischen Dosisfindungsstudie funktioniert, für eine vergleichende klinische Studie aus zwei verschiedenen interventionellen Verfahren und einem rein konservativen Vorgehen 
(manchmal auch als „Nichtstun“ fehlinterpretiert) ist ein derartiges Studiendesign ungeeignet. Während das BMT in SPACE-2 jetzt in den beiden Parallelstudien sehr gut untersucht werden wird, ist der „Head-tohead-Vergleich“ zwischen CAS und CEA leider aus finanziellen und logistischen Gründen innerhalb von SPACE-2 nicht mehr möglich. Man wird allerdings versuchen, mithilfe differenzierter statistischer Methoden (z. B. durch Anwendung eines „propensity score“) einen indirekten Vergleich herbeizuführen. Die Sorge um einen möglichen versteckten Selektions-Bias muss aber ernst genommen werden. Unabhängig von SPACE-2 können natürlich auch deutschsprachige Zentren an ACST-2 oder ECST-2 teilnehmen. Besonders ACST-2 schließt die durch das neue SPACE-2-Design entstandene Lücke des direkten Vergleichs zwischen CAS und CEA. Außerdem haben alle Studienleitungen (inkl. CREST-2) verabredet, ihre jeweiligen Daten zu „poolen“, um noch bessere Ergebnisse ermitteln zu können. Eine Teilnahme an einer oder an mehreren der genannten RCTs ist also absolut sinnvoll!

Werden die aktuell laufenden RCTs nun die Frage beantworten, welche Therapie (BMT allein, CEA +BMT oder $\mathrm{CAS}+\mathrm{BMT}$ ) die Beste ist für eine hochgradige asymptomatische Karotisstenose?

Die Antwort ist eindeutig JA, weil die geplante und begrüßenswerte Zusammenführung der Daten aller Studien zu wirklich großen Kollektiven führen wird, mit denen auch geringe Unterschiede hinsichtlich der prozeduralen Sicherheit und der Langzeitergebnisse statistisch belastbar detektiert werden können. Es ist diese Art von Evidenz, die der Gefäßchirurgie in den 1990er-Jahren (mit)entscheidend auf die Füße geholfen hat und dazu geführt hat, dass die CEA - gemessen am "Goldstandard RCTs“ - durch Studien wie NASCET, ECST, CREST, SPACE, ICSS, GALA, EV-3S u.v.a. zu Recht als eine der am besten untersuchten Operationsmethode überhaupt gepriesen wird. Diese großen RCTs haben allein und im Rah- men von Metaanalysen dazu geführt, dass die klinisch so wichtige Frage nach der optimalen Behandlung mittel- und hochgradiger symptomatischer Karotisstenosen auf allerhöchstem Evidenzniveau beantwortet werden konnte. Die CEA ist seither ein wichtiger Eckpfeiler in der Sekundärprävention der zerebralen Ischämie. Unser Fach hat davon sehr profitiert. Vermutlich wurde hierdurch so mancher Arbeitsplatz in der Gefäßchirurgie gesichert oder neu geschaffen!

Mein Appell richtet sich an alle Leser, eine Teilnahme an SPACE-2 und/oder einer der anderen europäischen randomisierten Studien (RCTs) wohlwollend zu prüfen. Es ist bekannt, dass Patienten innerhalb kontrollierter Studien nicht schlechter, sondern besser behandelt werden (Kontrolle der Risikofaktoren). Ihre Teilnahme an einer dieser Studien hilft also nicht nur unserer gemeinsamen Profession, sondern auch Ihren Patienten!

Nochmals sei allen Autoren für die umfassende Darstellung Ihrer Studien sehr herzlich gedankt.

Mit den besten Grüßen

Ihr

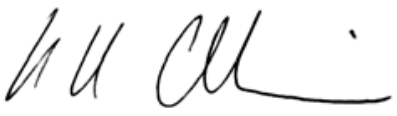

Prof. Dr. H.-H. Eckstein

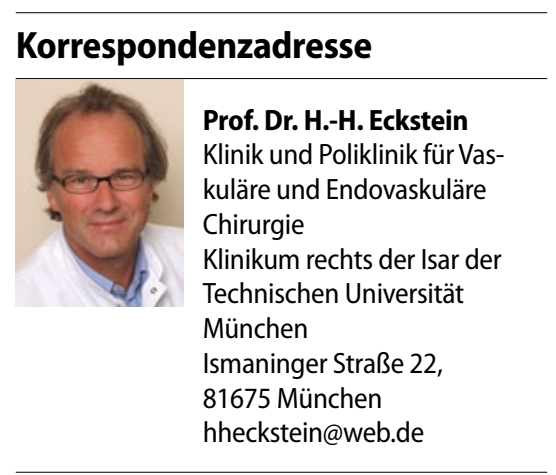

Interessenkonflikt. H.H. Eckstein gibt an, dass kein Interessenkonflikt besteht.

\section{Literatur}

1. Abbott AL. Medical (nonsurgical) intervention alone is now best for prevention of stroke associated with asymptomatic severe carotid stenosis: results of a systematic review and analysis. Stroke 2009;40:e573-e83.

2. Naylor AR, Rothwell PM, Bell PR. Overview of the principal results and secondary analyses from the European and North American randomised trials of endarterectomy for symptomatic carotid stenosis. EurJVascEndovascSurg 2003;26:115-29.

3. Ringleb P. S3- Leitlinie Extracranielle Carotisstenose, Kapitel 8.17: Welche Patienten sollten mit welcher Medikation konservativ behandelt werden? Gefässchirurgie 2012; 17(6):593-596.

4. Deutsch L, Haller B, Söllner H, Storck M, Eckstein $\mathrm{HH}$. Trends and results of carotid artery surgery in Germany 2003-2011. Part 1: Clinical stages, perioperative morbidity and mortality and assessment of quality indicators. Gefässchirurgie 2013, in press, DOI 10.1007/s00772-013-1231-8.

5. Ringleb P, Berkefeld J, Eckstein HH. S3- Leitlinie Extracranielle Carotisstenose, Kapitel 8.2: Wann und zu welchem Zeitpunkt besteht die Indikation zur OP oder zur Intervention einer asymptomatischen/ symptomatischen Stenose (einschließlich Notfallindikation), inkl. Subgruppen, die eher von einer operativen, endovaskulären oder konservativen Therapie profitieren? Gefässchirurgie 2012; 17(6):522-542. 